\title{
LA GÉNESIS DEL CONVENIO MULTILATERAL IBEROAMERICANO DE SEGURIDAD SOCIAL
}

FRANCISCO MANUEL JACOB SÁNCHEZ

Vicesecretario General de la Organización Iberoamericana de Seguridad Social 


\title{
RESUMEN
}

El Convenio Multilateral Iberoamericano de Seguridad Social constituye la culminación de una antigua aspiración en el seno de la Comunidad Iberoamericana.

Desde sus orígenes, el Convenio Multilateral ha estado íntimamente ligado a la Organización Iberoamericana de Seguridad Social (OISS). Precisamente fue en el congreso que ésta última organizó en el año 2004 donde surgió la idea de su elaboración que comienza a materializarse a partir de 2005 siendo aprobado el texto definitivo en 2007 durante la XVII Cumbre Iberoamericana de Jefes de Estado y de Gobierno celebrada en Santiago de Chile.

PALABRAS CLAVE: Convenio Multilateral Iberoamericano de Seguridad Social, Organización Iberoamericana de Seguridad Social, OISS, MERCOSUR.

\begin{abstract}
The Multilateral Ibero-American Agreement on Social Security is the culmination of a long-standing aspiration within the Ibero-American Community. Since its inception, the Multilateral Agreement has been closely linked to the Ibero-American Social Security Organization (ISSO). It was precisely at the congress that the latter organized in 2004 where the idea of its drafting arose. It began to materialize from 2005 and the final text was approved in 2007 during the XVII Ibero-American Summit of Heads of State and Government held in Santiago de Chile.
\end{abstract}

KEYWORDS: Ibero-American Multilateral Agreement on Social Security, IberoAmerican Social Security Organization, ISSO, MERCOSUR. 
SUMARIO

I. ANTECEDENTES

II. EL PROYECTO DE CMISS

III. EL PROCESO DE ELABORACIÓN

IV. EL ACUERDO DE APLICACIÓN DEL CMISS

V. LA ENTRADA EN VIGOR DEL CMISS

VI. CONCLUSIÓN 


\section{ANTECEDENTES}

El Convenio Multilateral Iberoamericano de Seguridad Social (CMISS) constituye la culminación de una antigua aspiración en el seno de la Comunidad Iberoamericana, cuyo primer antecedente probablemente se encuentra en el Convenio Iberoamericano de Seguridad Social, suscrito en Quito (Ecuador) en 1978. No obstante, este Convenio es, en realidad, un acuerdo marco que requiere para su entrada en vigor entre dos países que éstos suscriban, además, un acuerdo de aplicación complementario. Este camino ha sido efectivamente seguido en algunos casos, especialmente por Uruguay que prácticamente ha suscrito todos sus convenios de seguridad social con otros países iberoamericanos a través de esta técnica (y también en algún supuesto más como en el del convenio entre Argentina y Colombia).

Sin embargo, lo cierto es que aquél Convenio de 1978 no llegó a cumplir plenamente sus expectativas y por ello y como quedó plasmado en la Declaración de Bahía, aprobada con ocasión del XIII Congreso Iberoamericano de Seguridad Social, celebrado en el seno de la Organización Iberoamericana de Seguridad Social (OISS), en marzo de 2004, seguía viva esa aspiración de “... la mejora de las condiciones de vida de los ciudadanos, especialmente a través de la Seguridad Social, garantizando la adquisición y conservación de derechos en los movimientos migratorios, a través de Convenios Multilaterales de Seguridad Social que, a partir de los actualmente existentes o en fase de negociación, pudieran permitir en un futuro llegar a la adopción de un único Convenio Iberoamericano de Seguridad Social". A su vez, el CMISS encuentra también sus antecedentes en otros convenios previamente existentes. Tanto de carácter bilateral, a través de los más de cincuenta convenios vigentes entre países de la Comunidad Iberoamericana. Como en el ámbito multilateral, en el que las expresiones más recientes, en sus respectivos espacios de vigencia, serían el Acuerdo Multilateral de Seguridad Social del MERCOSUR, en aplicación desde 2008 y la Decisión 583, Instrumento Andino de Seguridad Social, aún pendiente de reglamentación, y consiguientemente sin vigencia por el momento. Así como en los Reglamentos Europeos 883/04 y 987/09 (en el momento de inicio de la andadura del CMISS los Reglamentos 1408/71 y 574/72), puesto que dos países de la Comunidad Iberoamericana, España y Portugal, forman también parte de la Unión Europea.

Pero a pesar de estos antecedentes, la cobertura que todos estos convenios bilaterales o multilaterales proporcionaban no llegaban a cubrir las necesidades de protección de un ámbito poblacional de más de 600 millones de personas que, además, integran la región del mundo en la que, según distintos Organismos Internacionales, más movimientos migratorios se producen, muchos de ellos entre países de esa misma región y para cuya completa cobertura serían necesarios más de 230 convenios bilaterales cruzados, de los que, en la realidad, no se dan más de un $25 \%$ que, además, se concentran esencialmente en cuatro de los 22 países de la Comunidad Iberoamericana, mientras que muchos otros no tenían vigente ningún convenio o un número mínimo de ellos, en muchos casos restringidos a ámbitos muy concretos como la atención sanitaria de urgencia y frecuentemente desconocidos y olvidados. 


\section{EL PROYECTO DE CMISS}

Así pues, ésta necesidad y los antecedentes citados son lo que llevan a que el proyecto de abordar un Convenio Multilateral Iberoamericano de Seguridad Social sea planteado por la Organización Iberoamericana de Seguridad Social (OISS) y acogido por XV Cumbre Iberoamericana de Jefes de Estado y de Gobierno (las Cumbres Iberoamericanas se vienen celebrando desde 1991, con carácter anual hasta 2014 y con periodicidad bianual a partir de ese año), celebrada en Salamanca (España), los días 14 y 15 de octubre de 2005 que, en su Declaración Final, en el apartado 17, adoptó la decisión de "iniciar el proceso de elaboración de un Convenio Iberoamericano de Seguridad Social con el objetivo de garantizar los derechos de Seguridad Social de los trabajadores migrantes y sus familias" y en el apartado 7 de dicha Declaración Final encarga a la Secretaría General Iberoamericana (SEGIB) la preparación de un Encuentro Iberoamericano sobre Migraciones que, en coordinación con la OISS, "apoye el proceso de preparación y suscripción de un Convenio Iberoamericano de Seguridad Social". Esta declaración supone el apoyo formal de la Cumbre Iberoamericana de Jefes de Estado y de Gobierno a los resultados obtenidos previamente en la V Conferencia Iberoamericana de Ministros y Máximos Responsables de Seguridad Social (con anterioridad a las respectivas Cumbres Iberoamericanas se convocan distintas conferencias ministeriales de carácter sectorial), celebrada los días 8 y 9 de septiembre de ese mismo año en Segovia (España), en cuyo Documento Final se acordó: "Iniciar el proceso de elaboración de un Convenio Multilateral de Seguridad Social de la Comunidad Iberoamericana....", en base a la consideración de que el proceso de mundialización y los diferentes movimientos de integración regional en curso conllevan, entre sus efectos, una mayor movilidad de las personas entre los diferentes Estados que debe ir acompañada de políticas sociales y económicas adecuadas, entre las que se encuentran la adopción de medidas de “... coordinación normativa en materia de protección social que, sin alterar los respectivos sistemas nacionales, permitan garantizar la igualdad de trato y los derechos adquiridos o en curso de adquisición de los trabajadores que se desplacen y de las personas de ellos dependientes".

Este mismo documento de la Conferencia de Segovia recoge ya una serie de parámetros y principios básicos que condicionan el contenido del proyecto: en primer término, el respeto las legislaciones nacionales que regulan los sistemas nacionales de Seguridad Social; también: "la igualdad de trato" a los nacionales de los países de la Comunidad Iberoamericana incluidos en el campo de aplicación personal del Convenio; la inclusión en su campo personal de aplicación tanto de los trabajadores que desarrollen una actividad dependiente o por cuenta ajena, como de los que la realicen de forma no dependiente o por cuenta propia, de forma legal, así como de sus familiares y sobrevivientes; la inclusión en el ámbito de aplicación material del Convenio de las prestaciones por vejez, invalidez, supervivencia y otras prestaciones económicas que pudieran derivarse de situaciones similares en función de las legislaciones nacionales respectivas, sin perjuicio de la posibilidad de ampliarlo a otras prestaciones; la determinación de la legislación aplicable, estableciendo como regla general la legislación nacional del país en el que se realiza la actividad laboral, sin perjuicio de las excepciones o de las reglas especiales que se determinen; la no derogación de los convenios bilaterales o multilaterales vigentes con anterioridad, aplicándose en caso de concurrencia las disposiciones más favorables; la garantía de los derechos en curso de 
adquisición a través de la totalización, si fuera necesaria, de los periodos de seguro, empleo o residencia (según el caso) cumplidos bajo la legislación de los diferentes Estados, sin perjuicio del cálculo de las prestaciones en proporción al tiempo efectivo de seguro, empleo o residencia en cada uno de los Estados ("prorrata temporis"); la garantía de los derechos adquiridos sin que éstos queden sometidos a reducción, suspensión, supresión o modificación por el hecho de haberse generado al amparo del Convenio, incluyendo su exportación; o la colaboración administrativa y técnica entre las instituciones de los diferentes Estados que facilite la tramitación y el reconocimiento.

Todos estos principios, habituales en los instrumentos de coordinación de legislaciones de seguridad social y señalados por la Conferencia Iberoamericana de Ministros y Máximos Responsables de la Seguridad Social, habían sido previamente debatidos y negociados durante varios meses a través de contactos e intercambio de documentos con los servicios técnicos de los distintos Estados, fruto de los cuales fueron, por ejemplo, la inclusión de los trabajadores por cuenta propia en el ámbito de aplicación del Convenio o la exclusión de las prestaciones sanitarias del mismo, por su tradicional dificultad de aplicación en este tipo de instrumentos.

No obstante, a pesar de quedar fijadas ya desde ese primer momento estas líneas generales, quedaba aún mucho camino por recorrer hasta lograr un texto consensuado y aceptado por los distintos Estados implicados en el proyecto y sus respectivos sistemas de seguridad social. En una zona con niveles de cobertura poblacional en protección social muy diferentes y en la que coexisten, en el ámbito de las prestaciones económicas, sistemas de reparto de corte clásico, con sistemas de capitalización individual, puros o mixtos y en el que conviven instituciones gestoras públicas con otras de carácter privado (Administradoras de Fondos de Pensiones, Administradoras de Riesgos Laborales, etc...).

Y Estados entre los que, por otra parte, no existe un vínculo político o jurídico que dote de unidad a la denominada Comunidad Iberoamericana, ni mecanismos de adopción de decisiones comunes con valor vinculante, más allá de las propias Declaraciones de las Cumbres y sus Conferencias Sectoriales, por lo que cualquier tipo de norma surgida de las actuaciones de estas instancias debe someterse al proceso de aprobación y ratificación propio de los tratados internacionales en cada uno de los países que conforman esa Comunidad.

\section{EL PROCESO DE ELABORACIÓN}

Así pues, desde esta perspectiva y a partir de los principios recogidos por la Conferencia de Ministros, fue necesario elaborar un primer documento que planteara diferentes cuestiones que, en base a tales principios, requerían una mayor precisión en sus planteamientos antes de proceder a elaborar un primer borrador de texto articulado del proyecto.

La primera de estas cuestiones fue la extensión del campo de aplicación personal del Convenio y, por consiguiente, del principio de "igualdad de trato", ya que aunque la Conferencia de Ministros sólo se refería los "nacionales" de los Estados Parte, cabía 
incluir otros supuestos como los nacionales de terceros países, refugiados o apátridas. Como es sabido, la decisión final, así recogida en el texto del Convenio fue la más generosa posible: "las personas que estén o hayan estado sujetas a la legislación de uno o de varios Estados Parte, así como a sus familiares beneficiarios y derechohabientes" (art. 2 del CMISS).

Otro tema a tratar fue el campo material de aplicación, sobre el que el documento de la Conferencia tampoco precisaba si se recogería una enumeración exhaustiva de las prestaciones a las que sería aplicable o si se debería proceder por la vía de la exclusión de aquéllas para las que no tendría aplicación. El criterio adoptado fue, en cierto modo mixto, al contemplar globalmente las prestaciones económicas contributivas de invalidez, vejez, supervivencia y las derivadas de accidentes de trabajo enfermedad profesional (sin entrar a enumerarlas) y excluir, también globalmente, las prestaciones médicas, los regímenes especiales o prestaciones exceptuados por los Estados Parte en los Anexos I y II, los regímenes no contributivos, la asistencia social y los establecidos a favor las víctimas de guerra. Y permitiendo la ampliación de este campo aplicación para dos o más Estados Parte cuando alcancen acuerdos al respecto que deberán ser inscritos en el Anexo III.

Del mismo modo era necesario precisar los términos de la vigencia de otros convenios, bilaterales o multilaterales a los que se refirió la Conferencia de Ministros y que, conforme a sus disposiciones, no deberían quedar derogados por el CMISS (éste fue y aún sigue siendo uno de los aspectos más debatidos en la negociación). A ello se refiere el artículo 8 del Convenio al establecer que en caso de concurrencia se aplicarán las "disposiciones" más favorables al beneficiario, así como que cada Estado Parte deberá proceder a informar los convenios bilaterales o multilaterales vigentes que quedarán registrados en el Anexo IV del CMISS.

Otra cuestión a debatir fue la determinación de la legislación de seguridad social aplicable y las excepciones a la norma general de aplicación de la legislación del lugar de trabajo, especialmente en el caso de traslado del trabajador por la empresa al territorio de otro Estado Parte y los plazos durante los que podría mantenerse la sujeción a la legislación del país de origen. Finalmente esta posibilidad quedó limitada a determinados colectivos de trabajadores cualificados y durante plazos relativamente cortos.

O la admisión, o no, de mecanismos de aseguramiento facultativo en el país de origen (que algunos Estados admiten) y su conciliación con el aseguramiento obligatorio en el país de trabajo. Así como el tratamiento de los periodos asimilados a los de cotización efectiva (el CMISS utiliza el término "periodos de seguro, de cotización o de empleo", de nuevo por las peculiaridades de los distintos países).

La consideración específica de los sistemas de pensiones de capitalización individual, muy extendidos en los países latinoamericanos, igualmente mereció un tratamiento individualizado, para resaltar la plena aplicación del Convenio también en estos supuestos y contemplar sus peculiaridades. 
Al igual que la forma de cálculo de las prestaciones causadas al amparo del Convenio y la aplicación de la totalización de períodos cotizados, así como si esta totalización debería hacerse en todo caso o sólo cuando el interesado no reuniera los requisitos necesarios para alcanzar el derecho a pensión considerando sólo los períodos acreditados en un Estado Parte.

Y, por último (aunque sin ánimo de exhaustividad) la constitución, o no, de órganos de apoyo, finalmente plasmada en la creación del "Comité Técnico Administrativo" y las cuestiones relativas a la firma, ratificación, entrada en vigor, etc... que no por ser de orden formal revisten menor importancia para la efectiva aplicación del Convenio

Todas estas cuestiones y sus distintas opciones fueron recogidas por la OISS en un documento denominado "Anteproyecto de Convenio Iberoamericano de Seguridad Social. Aspectos previos" que fue presentado ante representantes de 16 países iberoamericanos ya en febrero de 2006 y remitido a las autoridades de los 22 países de la Comunidad Iberoamericana entre marzo y julio de ese mismo año para, finalmente y en base al mismo y las observaciones recibidas, elaborar un primer borrador de texto articulado, nuevamente circulado y sometido a la I Reunión Técnica sobre el Convenio que tuvo lugar, en Montevideo (Uruguay), los días 10 y 11 de octubre de 2006, en la que se acordó proseguir con el proceso de consultas, sin perjuicio de declarar que el borrador "recoge fielmente los lineamientos y principios establecidos por el Documento Final de la V Conferencia Iberoamericana de Ministros/Máximos Responsables de Seguridad Social".

De acuerdo con ello, un segundo borrador de texto articulado del CMISS fue elaborado por la OISS y sometido a consulta en octubre de 2006 y, en base a las sugerencias y observaciones recibidas, un tercer borrador fue nuevamente circulado en marzo de 2007 y presentado a la II Reunión Técnica sobre el Convenio que tuvo lugar en Santiago de Chile los días 19 y 20 de abril de 2007.

En dicha Reunión quedaron cerrados buena parte de los artículos del Proyecto de Convenio, sin perjuicio de introducir modificaciones en otros, así como la inclusión de un nuevo título de "Clausulas Finales" que debería incorporarse al articulado y que dieron lugar a un nuevo borrador -el cuarto ya- que fue objeto de análisis en la III Reunión Técnica, celebrada en Sao Paulo (Brasil), los días 5 y 6 de junio de 2007, en la que, tras introducir algunas modificaciones más, se adoptó el acuerdo de "elevar el Proyecto de Convenio Multilateral Iberoamericano de Seguridad Social a la VI Conferencia Iberoamericana de Ministros/Máximos Responsables de la Seguridad Social, para su consideración y, en su caso, aprobación y presentación ante la XVII Cumbre Iberoamericana de Jefes de Estado y de Gobierno".

Esa VI Conferencia ministerial se celebró en Iquique (Chile), los días 5 y 6 de julio de 2007 y en ella los Ministros y/o Máximos Responsables de la Seguridad Social de 17 países iberoamericanos aprobaron, además de la declaración final de la Conferencia (denominada en esta ocasión "Declaración de Iquique"), una "Resolución sobre el Convenio Multilateral Iberoamericano de Seguridad Social", en la que se acuerda, en sus distintos apartados: "I. Aprobar el texto del Proyecto de Convenio Multilateral Iberoamericano de Seguridad Social que se acompaña como anexo, en idiomas español 
y portugués y que queda depositado en la Secretaría General Iberoamericana"; "II. Elevar el presente Acuerdo a la consideración de la XVII Cumbre Iberoamericana de Jefes de Estado y de Gobierno que tendrá lugar entre los días 8 y 10 de noviembre en Santiago de Chile"; "III. Someter a sus respectivos Gobiernos el texto aprobado, para su consideración y, en su caso, firma y ratificación conforme a las normas internas de cada país y su depósito en la Secretaría General Iberoamericana a través de la Organización Iberoamericana de seguridad Social (OISS), de acuerdo con lo dispuesto en el artículo 30 del mismo".

Quedó así cerrado el texto del CMISS en dicha reunión y en un tiempo inferior a dos años desde que, en la anterior V Conferencia, se adoptase la decisión de abordar este proyecto, plazo singularmente corto en comparación con otros instrumentos de coordinación de legislaciones de seguridad social.

Y, efectivamente, éste fue el texto que, elevado a la XVII Cumbre Iberoamericana de Santiago de Chile, reunida los días 8 a 10 de noviembre de 2007. fue adoptado por ésta en los siguientes términos: "...en cumplimiento de los acuerdos de las XV XVI Cumbres Iberoamericanas, los países miembros adoptan en esta ocasión el texto del Convenio Multilateral Iberoamericano de Seguridad Social y se comprometen a impulsar los procedimientos internos para su pronta entrada en vigencia. Así mismo acuerdan instruir a sus representantes técnicos para que inicien a la brevedad la negociación del Acuerdo de Aplicación".

Además, y por empeño personal de la Presidenta de Chile, en ese mismo acto 12 países procedieron a la firma del Convenio: Argentina, Bolivia, Brasil, Chile, Costa Rica, El Salvador, España, Paraguay, Perú, Portugal, Uruguay y Venezuela (posteriormente se incorporarían Colombia y Ecuador en 2008 y República Dominicana en 2011).

Quedó así completada en esa fecha, 10 de noviembre de 2007, la primera fase para la aplicación del CMISS y, a la vez y de forma inmediata, se dio inicio a la segunda fase: la negociación de su Acuerdo de Aplicación, conforme al mandato de la Cumbre.

\section{EL ACUERDO DE APLICACIÓN DEL CMISS}

El procedimiento seguido para esta negociación fue el mismo que anteriormente se había aplicado para el propio Convenio. Es decir, la preparación de sucesivos borradores por parte de la OISS -cinco en esta ocasión-, remitidos a autoridades y técnicos de todos los países para sus observaciones y sugerencias y la celebración, en este caso, de dos reuniones técnicas para avanzar y precisar los términos del Acuerdo.

Estas reuniones técnicas tuvieron lugar los días 18 y 19 de septiembre de 2008, en Fortaleza (Brasil) y los días 5 y 6 de marzo de 2009 en Santiago de Chile. En esta última quedo cerrado el texto, a nivel técnico y se acordó "Elevar, a través de la SEGIB y la OISS el Proyecto de Acuerdo de Aplicación del Convenio Multilateral Iberoamericano de Seguridad Social en idiomas español y portugués a una próxima VII Conferencia Iberoamericana de Ministros/Máximos Responsables de Seguridad Social para su consideración y, en su caso aprobación”. 
Finalmente, los días 10 y 11 de septiembre de 2009 la citada VII Conferencia acordó "Aprobar el texto del Acuerdo de Aplicación del Convenio Multilateral Iberoamericano de Seguridad Social, previsto en el artículo 26 del mismo, que se recoge como Anexo I, en idiomas español y portugués a un solo efecto y que quedará depositado en la Secretaría General Iberoamericana (SEGIB), a través de la Secretaría General de la OISS, conforme a lo dispuesto en el artículo 37 del Acuerdo". Así como "Elevar a sus respectivos Gobiernos el texto aprobado para su consideración y, en su caso y una vez ratificado el Convenio Multilateral Iberoamericano de Seguridad Social, su firma y depósito en la Secretaría General Iberoamericana a través de la Secretaría General de la OISS". Y "elevar el presente Acuerdo a la consideración de la XIX Cumbre Iberoamericana de Jefes de Estado y de Gobierno que tendrá lugar en Estoril (Portugal) los días 30 noviembre y 1 de diciembre de 2009". Cumbre que, a su vez, en el punto 22 de su Programa de Acción adoptó la decisión de "Dar seguimiento al proceso de ratificación del Convenio Multilateral Iberoamericano de Seguridad Social, suscrito en la XVII Cumbre Iberoamericana de Jefes de estado y de Gobierno, a fin de permitir su entrada en vigor y la firma del respectivo Acuerdo de Aplicación...". Quedando así cerrados los textos tanto del CMISS como de su Acuerdo de Aplicación y abierto el camino para su efectiva entrada en vigor.

\section{LA ENTRADA EN VIGOR DEL CMISS}

El propio Convenio, en su artículo 31 y su Acuerdo de Aplicación, en el artículo 33, establecen el procedimiento de entrada en vigor de ambos instrumentos. Procedimiento que, a su vez y dada la carencia de entidad jurídica de la Comunidad Iberoamericana, queda sujeto a los procesos internos de cada uno de los países.

En síntesis este procedimiento es el siguiente: firma del Convenio por el representante del Gobierno de cada Estado; ratificación parlamentaria del Convenio; depósito del instrumento de ratificación ante la Secretaría General Iberoamericana a través de la Organización Iberoamericana de Seguridad Social; firma del Acuerdo de Aplicación (generalmente por los Gobiernos, aunque en algún caso ha requerido una nueva ratificación parlamentaria); depósito del Acuerdo en la SEGIB a través de la OISS; y cumplimentación de los Anexos del Convenio y del Acuerdo de Aplicación.

Cumplidos estos trámites, el Convenio entraría en vigor "el primer día del tercer mes siguiente a la fecha en que se haya depositado el séptimo instrumento de ratificación, aceptación, aprobación o adhesión" (art.31.1), lo que tuvo lugar el día $1^{\circ}$ de mayo de 2011, primer día del tercer mes siguiente a la fecha de depósito del instrumento de ratificación por Bolivia (el séptimo), el 2 de febrero de 2011, tras haberlo hecho así con anterioridad El Salvador (4/9/2008), Chile (30/11/2009), Ecuador (4/11/2009), Brasil (11/12/2009), España (12/02/2010) y Portugal (22/12/2010).

A lo largo de ese mismo año 2011 y a medida que los respectivos países fueron suscribiendo el Acuerdo de Aplicación del Convenio, éste adquirió plena vigencia en Bolivia, Brasil, Chile, Ecuador, España, Paraguay y Uruguay. En 2012 lo hizo en El Salvador, en 2014 en Portugal y en 2016 en Argentina y Perú. En otros tres países firmantes, Colombia, Costa Rica y República Dominicana el CMISS se encuentra aún pendiente de ratificación parlamentaria y en distintos estados de tramitación. Mientras 
que Venezuela ya le ha ratificado pero no ha llegado a depositar el instrumento de ratificación.

Por último, en 2012 quedó también constituido el Comité Técnico Administrativo previsto en el Convenio y su Acuerdo de Aplicación, órgano esencial para el buen funcionamiento en la práctica de un instrumento que, deliberadamente, no se planteó como un texto amplio y detallado (al modo de los Reglamentos Europeos) sino más reducido y en cierto modo ambiguo, atribuyendo a este Comité, entre otras funciones, la de posibilitar la "aplicación uniforme" del Convenio. El Comité, presidido por el país correspondiente siguiendo un orden rotatorio alfabético anual, se ha reunido hasta la fecha en ocho ocasiones (tres en Uruguay, una en Bolivia, dos en Brasil, una en Chile y una en Ecuador), ha adoptado 20 "Decisiones", aprobado 10 formularios de uso denominados "IBERO 1-10", creado en su seno tres comisiones, elaborado un manual de procedimiento y puesto en marcha un sistema de transmisión electrónica de datos que se espera esté operativo en 2017.

\section{CONCLUSIÓN}

A lo largo del relato anterior se ha descrito, con cierto detalle, el proceso de gestación del Convenio Multilateral Iberoamericano desde sus antecedentes inmediatos hasta el momento actual en el que es una realidad en aplicación plena en 11 países iberoamericanos y a cuyo amparo se han tramitado ya más de 15.000 expedientes de pensiones en favor de ciudadanos migrantes o sus familias.

Como cabía esperar de la variedad de situaciones políticas, económicas y sociales y de la disparidad de los sistemas protectores existentes en los países implicados en el proyecto, no ha sido un camino fácil de recorrer. Existían dificultades de muy distinto tipo: idiomáticas (aunque las lenguas oficiales sean sólo dos, el español y el portugués, la variedad de acepciones y denominaciones es múltiple y en ambos idiomas); terminológicas; de fondo, dada la distinta naturaleza de los sistemas y las múltiples peculiaridades nacionales; de dispersión geográfica (22 países en dos continentes); de volatilidad institucional; y también, en ocasiones, de receptibilidad del proyecto por parte de algunos países o de sus autoridades en algunos momentos.

Pero, aun así, esta idea, nacida con tintes un tanto utópicos, fue acogida con interés y entusiasmo, por un nutrido grupo de personas y autoridades representativas de varios países, con cuyo apoyo y esfuerzo se logró lo que es hoy una realidad plena, con una amplia proyección de futuro que, cada vez de forma más tangible, contribuirá a mejorar las condiciones de vida de los ciudadanos que residan y trabajen en distintos países de la Comunidad Iberoamericana.

Porque la exclusión de los sistemas de seguridad social de los trabajadores migrantes o desplazados, derivada de la territorialidad de las leyes sociales, genera sin duda indefensión y desprotección para estas personas y sus familias, fomenta la informalidad y dificulta la movilidad internacional y, en su caso, el retorno a los países de origen. Y frente a estas cuestiones, instrumentos como el Convenio Multilateral Iberoamericano de Seguridad Social constituyen el principal medio para superarlas. 